\title{
SISTEMA DE INFORMAÇÕES GEOGRÁFICAS NO APOIO AO GERENCIAMENTO DE OBSTÁCULOS À SUPERFÍCIE DE SEGMENTO VISUAL
}

Geographic Information System to support managing of obstacles in Visual Segment Surface

\author{
FÁBIO ANDERSON BATISTA DOS SANTOS ${ }^{1}$ \\ CARLOS MÜLLER ${ }^{2}$
}

\author{
${ }^{1}$ Empresa Brasileira de Infraestrutura Aeroportuária - Infraero \\ Setor Comercial Sul, Quadra 04, Edifício Infraero \\ CEP: 70.304-902 - Brasília - DF - Brasil \\ ${ }^{2}$ Instituto Tecnológico de Aeronáutica - Departamento de Engenharia Civil \\ Praça Marechal do Ar Eduardo Gomes, 50, Vila das Acácias \\ CEP: 12.228-900 - São José dos Campos - SP - Brasil \\ fabsantos@infraero.gov.br; muller@ita.br
}

\section{RESUMO}

A segurança e a regularidade das operações em um aeroporto são influenciadas pelo uso do solo em seu entorno. De forma a preservar a segurança das operações de pouso por instrumentos nos aeroportos, a Organização Internacional de Aviação Civil instituiu uma superfície imaginária denominada Superfície de Segmento Visual (VSS). Qualquer objeto, natural ou artificial, que se estenda acima dessa superfície é considerado obstáculo. A existência de obstáculos pode gerar uma série de restrições, como o cancelamento do procedimento de aproximação por instrumento. O objetivo deste trabalho foi desenvolver uma metodologia para utilização de Sistema de Informações Geográfica (SIG) no apoio ao gerenciamento de obstáculos à VSS. Como estudo de caso, foi selecionadoo Aeroporto de Campo de Marte, em São Paulo, no qual a existência de obstáculos pode inviabilizar a implantação de procedimentos de aproximação por instrumentos. Foram utilizadas duas ferramentas complementares, uma ferramenta de SIG desktop e uma ferramenta de SIGWeb. A aplicação em SIG mostrou-se eficaz na análise dos obstáculos, mesmo sem utilizar um pacote de ferramentas para análise 3D. A 
aplicação foi suficiente ao integrar análise espacial em duas dimensões com uma consulta baseada em conceitos de geometria analítica.

Palavras-chave: SIG; Aeroporto; VSS; Obstáculos; Análise Espacial.

\begin{abstract}
The safety and regularity of airport operations are influenced by the landing use around it. In order to preserve safety of instrument landing operations at airports, the International Civil Aviation Organization (ICAO) has established an imaginary surface called Visual Segment Surface (VSS). Any object, natural or artificial which extends above the surface is considered an obstacle. Obstacles can generate a lot of restrictions, such as the cancellation of the instrument approach procedure. This study aims at developing a methodology for using Geographic Information System (GIS) to support management of obstacles in VSS. As a case study, Campo de Marte Airport, in São Paulo, was selected, in which the existence of obstacles can derail the implementation of instrument approach procedures. Two tools were used, a GIS desktop and a WEBGIS. The GIS application was effective in the analysis of obstacles, even without using a suite of tools for 3D analysis. The application was enough to integrate spatial analysis in two dimensions with a query based on analytical geometry concepts.
\end{abstract}

Keywords: GIS; Airport; VSS; Obstacles; Spatial Analysis.

\title{
1. INTRODUÇÃO
}

O crescimento da demanda por transporte aéreo no Brasil requer dos operadores aeroportuários investimentos em infraestrutura que garantam o atendimento a essa demanda a níveis de serviço aceitáveis. Dentre esses investimentos, podem ser citados: ampliação e ou construção de terminais de passageiros, construção de novas pistas de pouso e decolagem (PPD) e implantação de novos auxílios à navegação aérea. Além do atendimento à demanda, os operadores aeroportuários precisam atentar para a segurança das operações (pousos, decolagens, manobras no pátio, etc).

A segurança e a regularidade das operações em um aeroporto dependem da adequada manutenção de suas condições operacionais, que são diretamente influenciadas pelo uso do solo em seu entorno (BRASIL, 2011).

Uma construção no entorno de um aeroporto no prolongamento da PPD podecausar recuo da cabeceira da pista. Esse recuo de cabeceira acarreta perda de comprimento de pista disponível para pouso (Landing Distance Available -LDA). Por consequência, essa perda de LDA pode impedir que determinadas aeronaves operem naquele aeroporto ou que elas operem com sua capacidade máxima de passageiros e carga aérea.

De forma a preservar a segurança das operações de pouso por instrumentos nos aeroportos, a Organização Internacional de Aviação Civil (International Civil Aviation Organization - ICAO) instituiu uma superfície imaginária denominada 
Superfície de Segmento Visual (Visual Segment Surface - VSS). Qualquer objeto, natural ou artificial, que se estenda acima dessa superfície é considerado obstáculo.

Todos os novos procedimentos de aproximação direta por instrumentos, publicados a partir de 15 de março de 2007, devem ter suas VSS's livres de obstáculos.Além disso, os obstáculos existentes de procedimentos já publicados teriam que ser removidos ou rebaixados até 15 de março de 2012 (ICAO, 2006).

A existência de obstáculos à VSS pode gerar uma série de restrições, como o cancelamento do procedimento de aproximação por instrumento (DECEA, 2013). Esse cancelamento diminuiria a regularidade das operações, uma vez que as aeronaves passariam a operar apenas em condições meteorológicas favoráveis a pousos visuais.

Até 2012, o Departamento de Controle do Espaço Aéreo (DECEA) fornecia os dados das implantações que se configuravam obstáculos à VSS, para que os aeroportos realizassem as ações necessárias para remoção ou rebaixamento dos mesmos. A partir de 2013, os próprios aeroportos precisam realizar os levantamentos e indicar os obstáculos ao DECEA.

Nesse contexto, o objetivo deste trabalho foi desenvolver uma metodologia para utilização de Sistema de Informações Geográficas no apoio ao gerenciamento de obstáculos à VSS em empresas operadoras aeroportuárias. Como estudo de caso, foi realizado, através de SIG, análise espacial para gerenciamento dos obstáculos à VSS do Aeroporto de Campo de Marte. A metodologia desenvolvida permitiu analisar quais implantações (constantes de um cadastro) no entorno do aeroporto são ou não obstáculos à VSS.

\section{SUPERFÍCIE DE SEGMENTO VISUAL (VSS)}

A VSS é a fase do voo imediatamente após uma aeronave atingir condições visuais durante a realização de um procedimento de pouso por instrumento. Essa superfície depende das características do procedimento de pouso por instrumento (como ângulo de descida e desvio lateral em relação ao eixo da pista) e do código de pista. O código de pista está relacionado ao comprimento básico de pista da aeronave crítica que opera no aeroporto, conforme descrito em Brasil (2009).

Segundo ICAO (2006), os procedimentos de aproximação por instrumentos podem ser classificados em dois tipos:

- Aproximação de precisão (Precision Approach - PA) - procedimentos que utilizam orientação lateral e vertical providos por Sistema de Pouso por Instrumentos (Instrument Landing System - ILS) ou Radar de Aproximação de Precisão (Precision Approach Radar - PAR);

- Aproximação de não-precisão (Non-precision Approach - NPA) - são procedimentos de aproximação que proporcionam orientação lateral, mas nenhuma orientação vertical;

Medeiros (2011) define os procedimentos de aproximação de não-precisão como aqueles que fazem uso de ajudas de navegação em volta do aeroporto, como VOR (Very High Frequency Omni directional Range), DME (Distance Measuring 
Equipment) e NDB (Non-directional Beacon), para trazer a aeronave a um ponto onde a pista é visível, de forma que se consiga efetuar um pouso em condições visuais.

De acordo com o estudo, embora mais seguras que as aproximações de nãoprecisão, as aproximações com ILS possuem algumas limitações, como o alto custo de implantação e necessidade de grande espaço físico livre de obstáculos para sua instalação e operação.

ICAO (2008) define Navegação por Área (Area Navigation - RNAV) como método de navegação que permite que aeronaves operem em qualquer rota dentro da área de cobertura dos auxílios. Esse método remove a restrição imposta pelas rotas e procedimentos convencionais, nos quais a aeronave precisa sobrevoar os auxílios a navegação, permitindo flexibilidade operacional e maior eficiência.

Dentre as aproximações de não-precisão pode-se mencionar também as aproximações RNAV(GNSS - Global Navigation Satelite System). Esta aproximação é comumente chamada de LNAV (Lateral Navigation).

Uma das vantagens das aproximações RNAV (GNSS) é não precisar de equipamentos terrestres de rádionavegação. Existem procedimentos de aproximação de precisão que associam a orientação lateral dos procedimentos LNAV com orientação vertical fornecida a partir de altímetros barométricos e são comumente chamados de LNAV/VNAV (Vertical Navigation).

Segundo ICAO (2006), a VSS pode ser definida da seguinte forma:

a) Para procedimentos com localizer - instrumento de auxílio à aproximação de aeronaves que fornece dados sobre a direção da pista-ou que funcionem como localizer para referência de eixo, onde a trajetória final de aproximação estiver alinhada com o eixo da pista: superfície com largura da base igual à do início da Superfície de Aproximação, iniciando-se $60 \mathrm{~m}$ antes da cabeceira da pista e se estendendo paralelamente ao eixo da pista. A superfície termina no ponto onde sua altura atinge a Altura Livre de Obstáculos (Obstacle Clearence Height - OCH). A Figura 1apresenta a configuração da VSS para procedimentos com localizer.

Figura 1 - VSS para procedimentos de aproximação com localizer.

Fonte: ICAO (2006) modificado pelo autor.

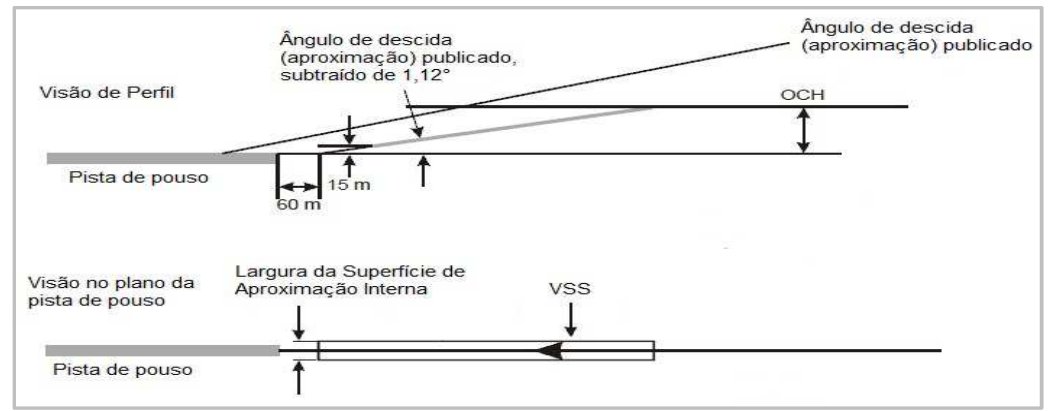

Bol. Ciênc. Geod., sec. Artigos, Curitiba, v. 20, nº 3, p.504-525, jul-set, 2014. 
b) Para todos os outros procedimentos de aproximação direta: largura da base de $300 \mathrm{~m}$ para pistas código 3 e 4 e de $150 \mathrm{~m}$ para pistas código 1 e 2 ; superfície origina-se a $60 \mathrm{~m}$ antes da cabeceira da pista, alargando-se com um coeficiente de $15 \%$ para cada lado do eixo da pista, e termina no ponto onde sua altura atinge o OCH.A Figura 2apresenta a configuração da VSS para procedimentos sem localizer.

Figura 2 - VSS para procedimentos de aproximação sem localizer. Fonte: ICAO (2006) modificado pelo autor.

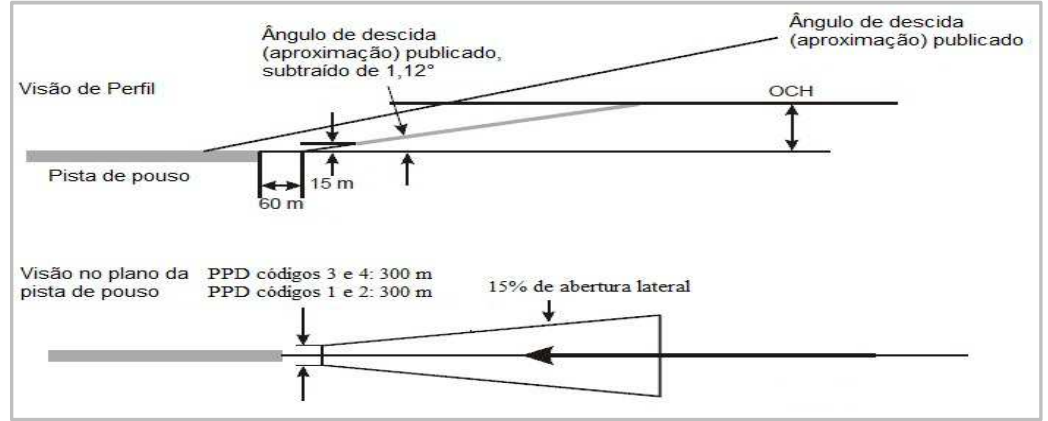

c) Quando o segmento da aproximação final for deslocado em relação ao eixo da pista e o interceptar, a largura no lado próximo ao curso de aproximação final será acrescido do ângulo de deslocamento em relação ao eixo. A Figura 3apresenta a configuração da VSS para esse tipo de procedimento.

Figura 3 - VSS para procedimentos de aproximação com desvio lateral em relação ao eixo da pista de pouso.

Fonte: ICAO (2006), modificado pelo autor.

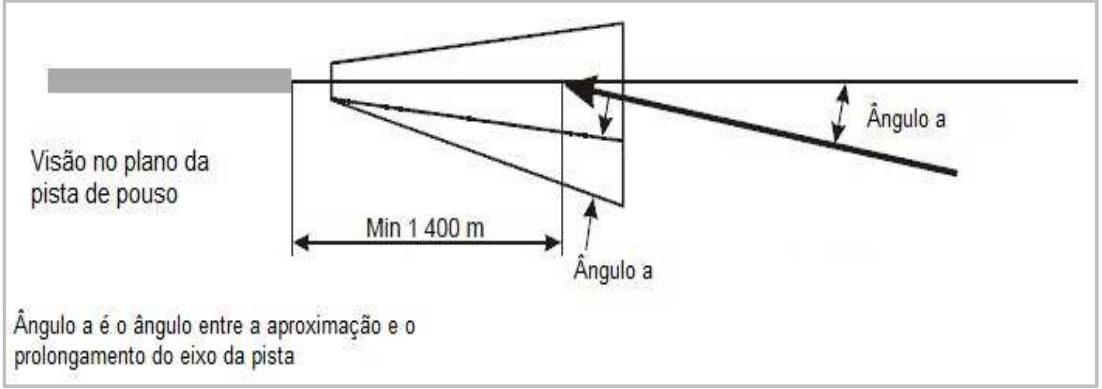

Bol. Ciênc. Geod., sec. Artigos, Curitiba, v. 20, nº 3, p.504-525, jul-set, 2014. 
Verticalmente, a VSS inicia-se na elevação da cabeceira e possui uma inclinação igual ao ângulo de descida do procedimento de aproximação menos $1,12^{\circ}$.

Se a VSS de algum procedimento de aproximação por instrumento for violada, ele não poderá ser publicado sem um estudo aeronáutico. A ação mitigadora resultado do Estudo Aeronáutico poderá definir, por exemplo, uma modificação do ângulo de descida e/ou um deslocamento da cabeceira.

Obstáculos que possuam altura inferior a $15 \mathrm{~m}$ acima da elevação da cabeceira podem ser desconsiderados. Além disso, obstáculos móveis, bem como aeronaves aguardando em ponto de espera da pista de pouso e decolagem são permitidos.

\section{OBSTÁCULOS EM AEROPORTOS}

Obstáculo é todo objeto de natureza permanente ou temporária, fixo ou móvel, ou parte dele, que esteja localizado em uma área destinada à movimentação de aeronaves no solo, ou que se estenda acima das superfícies destinadas à proteção das aeronaves em voo, ou ainda que esteja fora ou abaixo dessas superfícies definidas e cause efeito adverso à segurança ou regularidade das operações aéreas. (Brasil, 2011, p. 5)

A necessidade de os operadores aeroportuários identificarem obstáculos a superfícies limitadores previstas em normas traz à tona reflexão sobre a metodologia a ser utilizada. A metodologiatradicionalmente utilizada pelo DECEA para identificação de obstáculos nos aeroportos brasileiros foilevantamento topográfico convencional.

De acordo com Parish \& Nowak (2009), a metodologia tradicionalmente utilizada para detecção de obstáculos em aeroportos dos Estados Unidos consiste em uma combinação de fotogrametria com topografia convencional.

De acordo com os autores, esse método demonstrou-se preciso e confiável ao longo dos últimos cinquenta anos. Entretanto, o desejo de aumentar a eficiência e reduzir os custos dos levantamentos tem levado ao crescimento do interesse na tecnologia LIDAR(Light Detection and Ranging).

Parish (2003) analisou a habilidade de se melhorar a detecção de obstáculos em aeroportos a partir de tecnologia LIDAR pela modificação de alguns parâmetros do levantamento. Os principais parâmetros investigados foram ângulo de inclinação do voo, altura do voo e base do laser, embora parâmetros como velocidade do voo, sensibilidade do receptor também tenham sido discutidos.

Segundo o estudo, em trabalhos anteriores, mostrou-se que muitos obstáculos às operações em aeroportos, particularmente postes, antenas e outros objetos com diâmetros pequenos, foram geralmente não detectados pelos levantamentos LIDAR.

Foram apresentados resultados de voos sobre o Aeroporto Regional de Gainesville, Flórida, utilizando quatorze diferentes configurações de coleta de dados. Esses dados coletados por LIDAR foram comparados com dados de levantamentos de obstáculos realizados por topografia convencional, obtidos da National Geodesic Survey (NGS).

Bol. Ciênc. Geod., sec. Artigos, Curitiba, v. 20, nº 3, p.504-525, jul-set, 2014. 
A análise dos dados mostrou que melhorias significativas na detecção de obstáculos podem ser alcançadas com configurações adequadas. Foi mostrado também que uma detecção de $100 \%$ dos obstáculos pode ser alcançada com levantamentos LIDAR, com uma precisão vertical menor que um metro.

Martins (2010) desenvolveu uma metodologia para o uso de imagens de satélite de alta resolução espacial no gerenciamento de zonas de proteção de aeródromos. Como estudo de caso, foram analisados, com o uso de ferramenta SIG, os obstáculos à zona de proteção do Aeroporto Internacional Salgado Filho.As coordenadas dos obstáculos foram obtidas com base em Brasil (2005) e em levantamento topográfico realizado pelo Instituto de Cartografia Aeronáutica (ICA) em setembro de 2008.

Não foi realizada análise para verificar quais implantações no entorno do aeroporto eram ou não obstáculos. A ferramenta utilizada serviu apenas para a visualização de um cadastro de obstáculos pré-existente sobre a imagem de satélite de alta resolução espacial.

Como critérios da especificação técnica para aquisição da imagem foram considerados: tamanho máximo do pixel de $1 \mathrm{~m}$; resolução radiométrica mínima de 8 Bits;cobertura máxima de nuvens de $5 \%$ e data da imagem (imageamento) de no máximo 4 meses.

Como apoio de campo para realização das correções geométricas na imagem, foi realizado levantamento topográfico de campo com uso de GPS para coleta de pontos de controle. Foi adotada técnica de posicionamento relativo para a coleta das coordenadas dos pontos. O georreferenciamento e a ortorretificação foram realizados em conjunto, com auxílio do Software RSI ENVI.

Como conclusão do trabalho, destaca-se que o uso de imagens de satélite de alta resolução espacial apresentou-se como uma técnica eficiente para a análise de obstáculos, uma vez que o erro estimado nas coordenadas da imagem ortorretificada é tolerável na maior parte dos parâmetros estabelecidos pelas superfícies limitadoras.

\section{APLICAÇÃO DE SISTEMAS DE INFORMAÇÃO GEOGRÁFICA EM AEROPORTOS}

O uso de ferramentas de SIG para aeroportos tem se difundido nos últimos anos. Jia et. al (2004) desenvolveram um sistema baseado em SIG denominado ARO (Airport Runway Optimization) que determina a melhor orientação de uma pista de pouso e decolagem.

De acordo com o estudo, a determinação da orientação de uma PPD é uma tarefa crítica no planejamento aeroportuário. A direção da pista influencia as outras facilidades existentes no aeroporto, como terminais de passageiros, configuração das pistas de taxi e dos pátios de manobras, áreas de circulação, etc.

Um método de rosa dos ventos baseado em SIG foi apresentado para a determinação da melhor orientação de uma PPD. Esse método considerou uma rosa dos ventos como um banco de dados SIG em que cada célula é tratada como um 
polígono. Um único identificador é atribuído a cada polígono para representar a direção e a velocidade do vento.

Ainda de acordo com Jia et. al (2004), esse método beneficia-se de funções de SIG para lidar com o problema de cobertura parcial. Esse problema está associado à dificuldade de se calcular o percentual de ocorrência de ventosquando uma ou mais células da rosa dos ventos tradicional apresentam cobertura parcial. Dessa forma, ao formular uma rosa dos ventos como banco de dados de um SIG, evitam-se os cálculos geométricos computacionais.

Zimmer et. al. (2007) apresentaram uma nova possibilidade para a geração de imagens de familiarização aeroportuárias atravésde SIG. Dentre os dados que compõem o SIG, podem-se citar imagens de sensoriamento remoto, dados vetoriaisde aeroportos, cadastro de obstáculos e informações sobre procedimentos de aproximação.

De acordo com o estudo, nos Estados Unidos, uma qualificação aeroportuária é necessária para que um piloto possa executar procedimentos de aproximação e decolagens em aeroportos considerados "desafiadores" (challenging airports).Essa qualificação da qual os pilotos precisam pode ser obtida por meio das chamadas imagens de familiarização aeroportuária ou a partir de um voo de familiarização juntocom uma pessoa autorizada.

Santos (2012) desenvolveu um Sistema de Informação Geográfica de Participação Pública (PPGIS -Public Participatory Geographic Information System) com o objetivo de avaliar as contribuições que a população afetada pelo ruído aeronáutico podem oferecer na gestão desse impacto.

Foi desenvolvido um modelo de PPGIS para o Aeroporto de São José dos Campos em ambiente web, por meio de programas livres de licença. Usuários residentes nas proximidades do aeroporto foram estimulados a participar.

Dentre os resultados da pesquisa realizada, destaca-se que muitos usuários residentes em áreas fora dos limites do Plano Específico de Zoneamento de Ruído (PEZR) indicaram nível alto de incômodo ao ruído aeronáutico. Como explicação para esse fato, foi mencionada a possibilidade de o PEZR estar desatualizado.

Huang et. al. (2012) desenvolveram uma ferramenta de SIG na web que permite ao usuário explorar as relações entre as distribuições globais de doenças infecciosas transmissíveis, como malária, dengue e febre-amarela, com as rotas aéreas internacionais, com o objetivo de prover evidencias que ajudem a criar estratégias de mitigação para a importação (disseminação) dessas doenças através do transporte aéreo.

Segundo o estudo, o transporte aéreo modificou o cenário epidemiológico mundial, ao prover rotas de um lado para outro do planeta que podem ser utilizadas por pessoas infectadas com uma duração significativamente menor que o tempo de incubação da maioria das doenças infecciosas. A ferramenta desenvolvida foi denominada "Vector-borne disease airline importation risk" - VDB-AIR (Risco de importação de doenças transmissíveis por meio de transporte aéreo). 
Bentes et. al (2013), através do uso de simulação, modelos integrados de ruído e SIG, quantificarama proporção da população exposta a altos níveis de ruído aeronáutico no entorno do Aeroporto Internacional de Viracopos em Campinas.

Para a elaboração das curvas de ruído foi utilizado o software INM, um modelo computacional desenvolvido pela Federal Aviation Administration (FAA) que avalia o impacto do ruído aeronáutico na vizinhança dos aeroportos. Esse modelo é baseado em um algoritmo da Society of Automotive Engineers(Sociedade dos Engenheiros Automotivos).

Com utilização de software de SIG, as curvas de ruído foram associadas a dados censitários de população, o que possibilitou a determinação da quantidade de pessoas dentro de cada curva.

Sanches et. al (2014) associaram uso de software de SIG convencionalcom informações de uma base de dados pública para mensurar a população dentro da área de influência de cada aeroporto da União Europeia que opera voos regulares.

A base de dados utilizadaé denominada "CORINE", proveniente da Agência Europeia de Meio Ambiente e fornece informação sobre a estimativa de densidade populacional dos 27 países da União Europeia, mais Croácia e Moldávia, com uma resolução espacial (pixel) de 1 ha. Esse nível de detalhe da densidade populacional é bem maior que os das informações utilizadas em estudos anteriores.

Através da ferramenta ArcGis 9.3 foi calculado o número de habitantes dentro de raios de distância de $25 \mathrm{~km}, 50 \mathrm{~km}$ e $100 \mathrm{~km}$ de cada um dos 459 aeroportos que operavam voos regulares em 2009.

\section{ESTUDO DE CASO: AEROPORTO DE CAMPO DE MARTE}

O Aeroporto de São Paulo - Campo de Marte (SBMT) está localizado na Zona Norte da cidade de São Paulo, próximo ao terminal rodoviário do Tietê, à estação de metrô e da Marginal Tietê, via de acesso a rodovias estaduais e interestaduais.

O SBMT é o quinto maior aeroporto brasileiro em termos de movimentação de aeronaves (número de pousos mais número de decolagens), conforme se pode observar na Figura 4:

Não há operação de voos regulares no SBMT, mas ele pode ser considerado o maior aeroporto de aviaçãonão-comercial do país. O aeroporto opera voos de aviação geral, executiva, taxi aéreo e voos de instrução (aeroclube).Entre os anos de 2006 e 2012, o movimento operacional do SBMT cresceu, em média, 9,12\% ao ano.

De acordo com Infraero (2013), cerca de 70\% das operações no SBMT são de aeronaves de asa rotativa (helicópteros) enquanto os outros $30 \%$ representam operação com aeronaves de asa fixa.

O SBMT não possui procedimento de aproximação por instrumento publicado. Entretanto, de forma a aumentar a segurança da aproximação, planeja-se a publicação de procedimentos RNAV (LNAV e LNAV/VNAV) para as duas cabeceiras. 
Figura 4 - Gráfico do Movimento Operacional Anualdos 10 aeroportos mais movimentados do país, nos últimos dois anos.

Fonte: Infraero (2014).

Legenda:
SBGR Aeroporto Internacional de São Paulo - Guarulhos
SBSP: Aeroporto de Congonhas
SBBR: Aeroporto Internacional de Brasília
SBGL: Aeroporto Internacional do Rio de Janeiro - Galeão
SBMT: Aeroporto de Campo de Marte
SBRJ: Aeroporto Santos Dumont
SBKP: Aeroporto Internacional de Campinas - Viracopos
SBCF: Aeroporto Internacional de Belo Horizonte - Confins
SBSV: Aeroporto Internacional de Salvador
SBPA: Aeroporto Internacional de Porto Alegre
SBD

Conforme mencionado, a VSS se estende a partir de $60 \mathrm{~m}$ além da cabeceira, comum ângulo de inclinação vertical igual ao ângulo do procedimento de aproximação subtraído de $1,12^{\circ}$, até alcançar a $\mathrm{OCH}$.

$\mathrm{O}$ ângulo de descida padrão para procedimentos de aproximação por instrumentos no Brasil é de $3^{\circ}$, portanto, será considerada VSS do SBMT com inclinação vertical de $1,88^{\circ}$.

A OCH deve ser publicada para cada procedimento de aproximação. Como os procedimentos RNAV do SBMT ainda não foram publicados, neste trabalho será considerado $\mathrm{OCH}$ igual ao limite inferior possível, de $115 \mathrm{~m}$ acima da elevação da cabeceira para aeronaves categoria B, conforme ICAO (2006). A Figura 5apresenta esquema das VSS do SBMT: 
Figura 5 - Esquema da VSS da cabeceira 30 do SBMT. Imagem Google 3D.

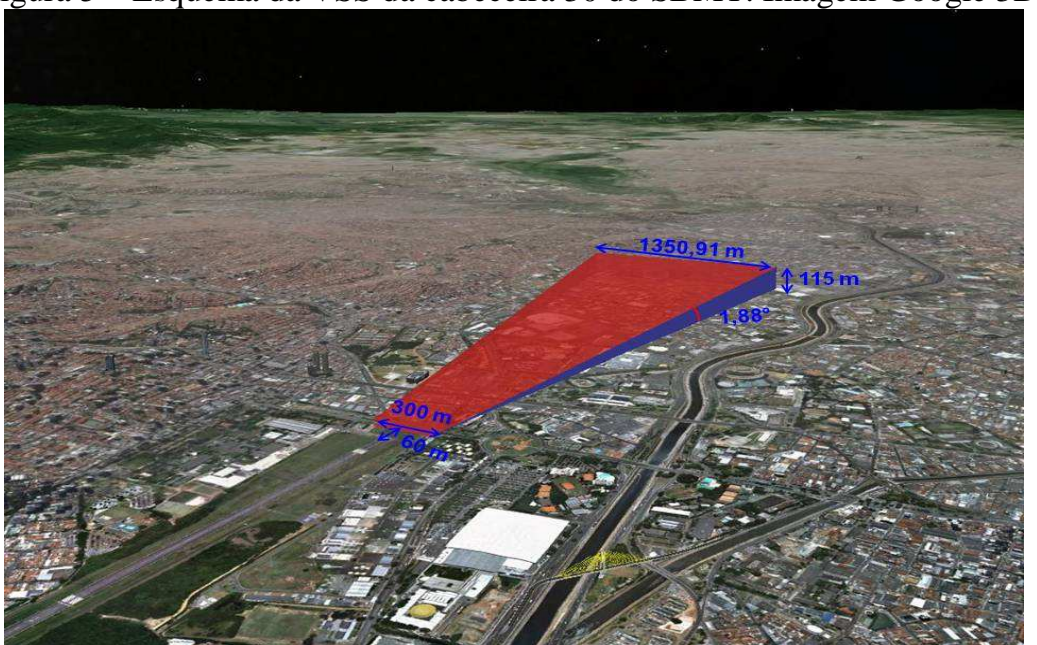

\section{METODOLOGIA E RESULTADOS}

No presente trabalho foram utilizadas duas alternativas complementares de SIG, O SIG Desktop e um Servidor Web de Dados Geográficos (SIG Web). Como ferramenta Desktop foi utilizado o software ArcGis Desktop (ArcEditor) 10.1. Como ferramenta Web foi utilizado o ArcGis Server. As licenças dos softwares utilizados são de propriedade da Infraero. A Figura 6 apresenta a metodologia de desenvolvimento da aplicaçãoem SIG:

Figura 6 - Metodologia Desenvolvimento da aplicação em SIG.

\begin{tabular}{|c|c|}
\hline ETAP & •Descrição dos dados \\
\hline ETAP & - Criação de conjuntos de dados de classes de feições \\
\hline ETAP & - Preenchimento das tabelas de atributos das classes de feições \\
\hline ETAP & - Publicação dos dados \\
\hline ETAP & - Gerenciamento da Aplicação WEB \\
\hline
\end{tabular}

Como o objetivo da aplicação SIG deste trabalho é permitir a diversos usuários visualização, pesquisa, edição do banco de dados e análise espacial, torna-se custosa a aquisição de diversas licenças de uma ferramenta de SIG Desktop. Nesse sentido, uma solução de menor custo é a aquisição de uma ferramenta de SIG Web. 


\subsection{Descrição dos Dados}

As informações inseridas no SIG estão representadas na Figura 7 na forma de camadas (layers). Todos os dados inseridos no SIG encontram-se no Sistema Geodésico de Referência WGS-84.

Figura 7: Informações constantes do SIG. Inspirado em Martins (2010).

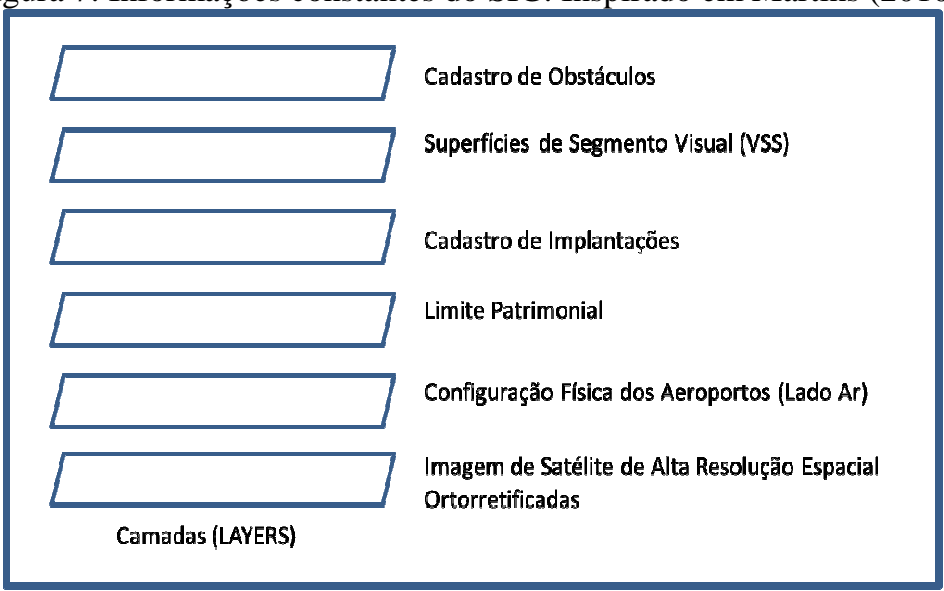

Analisando a Figura 7, a imagem de satélite utilizada foi adquirida pela Infraero e ortorretificada pela equipe responsável por geoprocessamento dentro da Empresa. A imagem é do sensor Pleiades de agosto de 2012, com $50 \mathrm{~cm}$ de resolução espacial, 11 Bits de resolução radiométrica e abrange uma área de $60 \mathrm{~km}^{2}$.

A configuração física do lado ar (parte do aeroporto onde há movimentação de aeronaves)do SBMTfoi elaborada através de Ferramenta CAD com base nas coordenadas das cabeceiras da PPD, constantes de levantamentos realizados pelo ICA em 01/07/2009.

O limite patrimonial foi elaborado através de Ferramenta CAD com base em levantamento realizado pelo ICA em 01/07/2009.

O cadastro de implantações tem como fonte quatro levantamentos topográficos realizados peloICA, respectivamente em 01/07/2007, 01/07/2009, 20/09/2010 e 13/08/2012. O Datum vertical é o de Imbituba.

Dentre as informações que constam do levantamento destacam-se: descrição da implantação (árvore, poste, antena, edificação, etc), coordenadas geográficas e elevação.

A Superfície de Segmento Visual foi elaborada com base nas coordenadas da PPD, considerando ICAO (2006).

O cadastro de obstáculos à VSS foi produzido com base nas VSS's elaboradas e no cadastro de implantações, através de análise espacial. Esse processo será descrito na seção 6.6 . 


\subsection{Criação de Conjuntos de Dados de Classes de Feições}

Primeiramente, os dados foram importados por meio da Ferramenta de SIG Desktop (ArcEditor). Em seguida, com base nos dados, foram criadas as classes de feições.

Uma classe de feição (Feature Class) é uma coleção de feições geográficas (objetos) que compartilham uma mesma geometria, os mesmos atributos e a mesma referência espacial, como por exemplo:

- Classe de feição de auxílios à navegação aérea - geometria: pontos; atributos: nome, tipo, elevação, etc;

- Classe de feiçãode pistas de pouso e decolagem - geometria: polígono ou linhas; atributos: orientação, comprimento, largura, etc.

Um conjunto de dados de feições (Feature Dataset) é composto de classes de feições que foram agrupadas de forma que elas possam participar em relacionamentos topológicos umas com as outras.

Todas as classes de feição em um Feature Dataset devem compartilhar a mesma referência espacial, isto é, elas devem ter o mesmo sistema de coordenadas e suas feições devem estar dentro de uma área geográfica comum. Nesse sentido, foi criado um Feature Dataset que agrupou todas as classes de feição do SBMT.

\subsection{Preenchimento das Tabelas de Atributos das Classes de Feições}

Após a criação do Feature Dataset, é necessário que as tabelas de atributos das classes de feições sejam modificadas, de forma que apresentem as informações desejadas.

Quando as geometrias são desenhadas em Ferramenta CAD e exportadas para classe de feição, apresentam a informação de forma e localização, mas não outros atributos que possam ser julgados relevantes.

No caso de uma classe de feição de pista de pouso, por exemplo, é necessária a criação/preenchimento de atributos como comprimento, largura, orientação, distâncias declaradas, etc.

No caso de uma faixa de pista, é necessária a criação/preenchimento de atributos como a que pista ela está associada e suas dimensões.

\subsection{Publicação dos Dados}

Após a criação do conjunto de dados de feições (Feature Dataset) e o preenchimento das tabelas de atributos, um arquivo de mapa contendo as classes de feições e a imagem de satélite foi elaborado.

Dentre as atividades relacionadas à criação do arquivo de mapa destacam-se a criação de simbologia (aparência) das classes de feições bem como a definição de que classes de feição serão visíveis pelos usuários a cada nível de zoom.

Após o processo de sua criação, o arquivo de mapa foi publicado no Servidor de SIG Web (ArcGIS Server). A Figura 8apresenta o arquivo de mapa do SBMT publicado: 
Figura 8 - Mapa do SBMT com as informações publicadas no SIG.

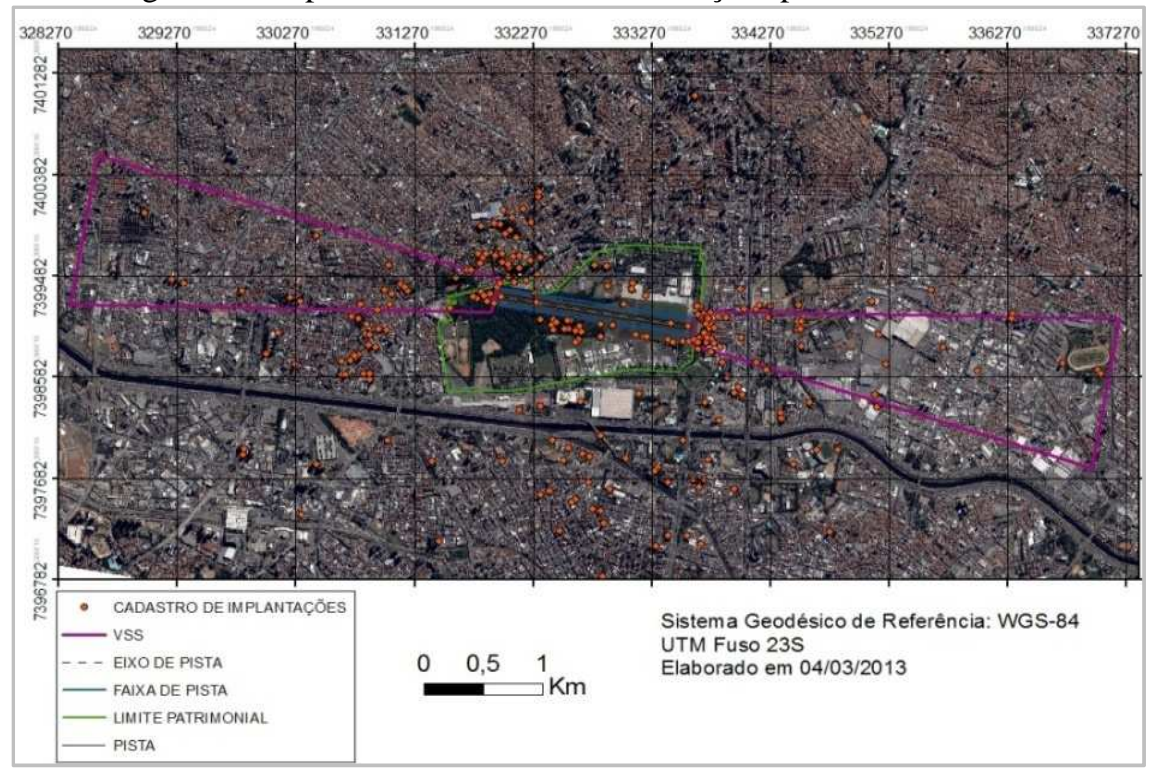

\subsection{Gerenciamento da Aplicação WEB}

A aplicação web faz-se necessária, pois é a ferramenta que permite,a utilização dos dados publicados no Servidor de SIG WEB pelos usuários. Essa utilização dos dados é feita por meio da internet. A aplicação WEB utilizada no presente trabalho foi adquirida pela Infraero juntamente com o Software ArcGIS Server.

Através da aplicação WEB é possível definir quais classes de feições, nesse contexto, chamadas de camadas, são acessíveis a cada tipo de usuário. Além disso, é possível definir quais ferramentas são acessíveis para cada tipo de usuário.Dentre as ferramentas da aplicação web utilizada nesse trabalho destacam-se:

- Consulta - permite a consulta de atributos de classes de feições por meio do simples passar do mouse sobre a geometria;

- Pesquisa avançada com base nos atributos das camadas (classes de feições);

- Geração de mapas temáticos.

Como exemplos de pesquisas avançadas que podem ser realizada pela aplicação web, podem-se citar:

- quantas implantações em torno do SBMT possuem elevação de topo maiores que determinado valor?

- quantos obstáculos à VSS no SBMT são árvores (teoricamente mais fáceis de se rebaixar/retirar? Quantos são torres de celulares e quantos são prédios? 


\subsection{Análise dos Obstáculos à VSS do SBMT}

O ArcGIS Desktop e a aplicação WEB utilizada no presente trabalho não são capazes de realizar análises em 3D por não possuir o pacote de ferramentas necessário.

Diante disso, com o objetivo de se avaliar quais implantações constantes do cadastro são obstáculos à VSS do SBMT, foi utilizada a seguinte metodologia, apresentada na Figura 9.

Figura 9 - Metodologia para determinação dos obstáculos à VSS do SBMT.

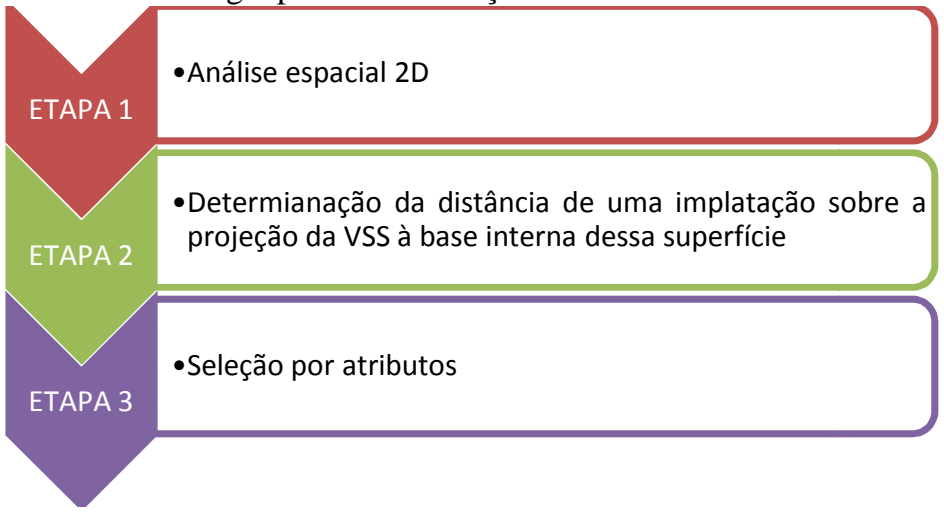

\subsection{Análise Espacial 2D}

A primeira etapa consiste de uma análise espacial 2D que retorne quais implantações constantes do cadastro encontram-se sobre a projeção horizontal (no plano xy) da VSS, para as duas cabeceiras separadamente.Essa etapa foi realizada através de ferramenta de interseçãoentre as camadas de cadastro de implantações e da VSS, denominada intersect.

A Figura 10apresenta o resultado dessa análise espacial. Das 281 implantações constantes do cadastro e já inseridas no SIG, após análise espacial 2D, verificou-se a existência de 26 implantações sob a projeção da VSS do procedimento de aproximação da cabeceira 12 e 84 implantações sob a projeção da VSS do procedimento de aproximação da cabeceira 30.

\subsection{Distância da Implantação à Base da VSS}

Para se analisar se uma implantação sobre a projeção da VSS é obstáculo ou não, precisa-se determinar a distância (perpendicular e no plano xy) entre a implantação e a base da VSS. Com base nessa distância e em parâmetros como elevação da cabeceira, elevação de topo da implantação e ângulo de inclinação da VSS, pode-se determinar se o topo se uma implantação ultrapassa a referida superfície. 
Figura 10 - Resultado da Análise Espacial 2D.

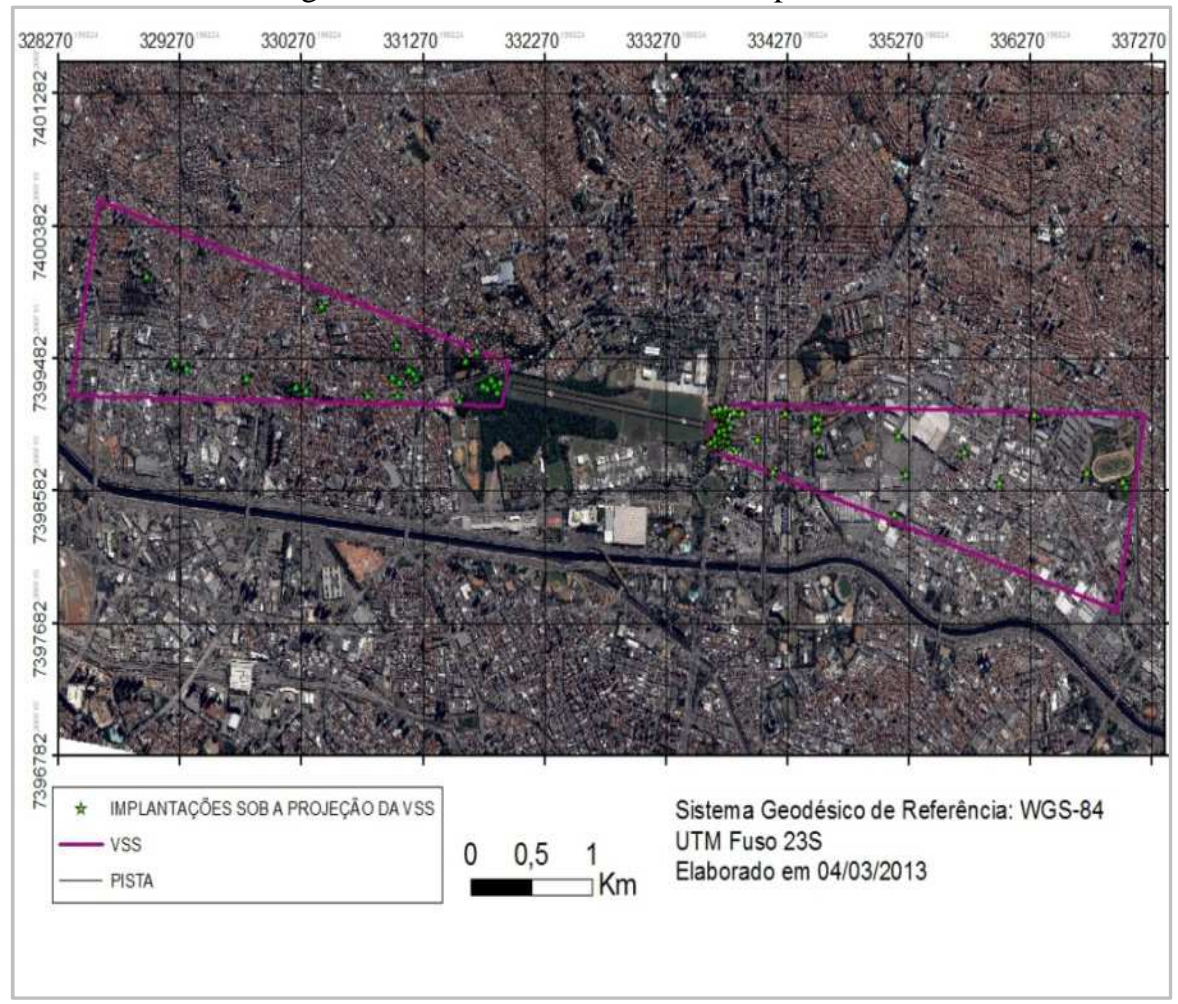

Conforme mencionado, para o caso de Aeroporto Código 3 e 4, como é o caso do SBMT, a base da VSS possui largura de $300 \mathrm{~m}$ e está localizada a 60 da cabeceira. A base da VSS é perpendicular ao eixo da pista.

Nesse sentido, faz-se necessário determinar a equação da reta que passa pelo ponto a $60 \mathrm{~m}$ da cabeceira, no prolongamento do eixo da pista de pouso, e que seja perpendicular a esse eixo. Para a determinação da equação da reta perpendicular ao eixo da pista de pouso, faz-se necessário determinar primeiro a equação da reta que define o eixo da pista.

Sejam CAB12 ( $\left.\mathrm{X}_{\mathrm{cab12}}, \mathrm{Y}_{\mathrm{cab} 12}\right)$ e CAB30 $\left(\mathrm{X}_{\mathrm{cab30}}, \mathrm{Y}_{\mathrm{cab30}}\right)$, os pontos que representam as cabeceiras da pista, onde $\mathrm{X}$ e $\mathrm{Y}$ representam as coordenadas este $\mathrm{e}$ norte, respectivamente. A equação da reta que passa por esses dois pontos (eixo da pista de pouso) pode ser descrita em (1):

$$
y=\frac{\left(Y_{c a b 30}-Y_{c a b 12}\right)}{\left(X_{c a b 30}-X_{c a b 12}\right)} \cdot x+\frac{\left(X_{c a b 30} \cdot Y_{c a b 12}-X_{c a b 12} \cdot Y_{c a b 30}\right)}{\left(X_{c a b 30}-X_{c a b 12}\right)}
$$


Substituindo os valores das coordenadas x(este) e y(norte) das cabeceiras, temos a seguinte equação do eixo da pista de pouso do SBMT:

$$
y=-0,169373 . x+7.455 .531,626
$$

A equação que define as retas perpendiculares ao eixo da pista é escrita conforme(3):

$$
y=\frac{-1}{-0,169373} \cdot x+c \rightarrow y=5,904133 \cdot x+c
$$

Considerando a existência de VSS para os procedimentos a partir das duas cabeceiras e que a base das superfícies encontra-se a $60 \mathrm{~m}$ no prolongamento do eixo da pista de pouso e decolagem, as equações das retas que passam pelas bases das VSS estão apresentadas em(4) e (5):

$$
\begin{aligned}
& \mathrm{y}_{\text {base_vss_cab12 }}=5,904133 \cdot \mathrm{x}+5.439 .473,556 \\
& \mathrm{y}_{\text {base_vss_cab30 }}=5,904133 \cdot \mathrm{x}+5.429 .177,336
\end{aligned}
$$

Seja "a.x + b.y + c" a equação geral de uma reta s e $\mathrm{P}\left(\mathrm{x}_{0}, \mathrm{y}_{0}\right)$ um ponto qualquer. A distância (perpendicular)entre o ponto $\mathrm{P}$ e a reta s pode ser expresso de acordo com (6):

$$
\mathbf{d}=\frac{\left|\mathbf{a} \cdot \mathbf{x}_{0}+\mathbf{b} \cdot \mathbf{y}_{0}+\mathbf{c}\right|}{\sqrt{\mathbf{a}^{2}+\mathbf{b}^{2}}}
$$

Com base em (4), (5) e (6), pode-se definir a distância entre uma implantação $\mathrm{P}(\mathrm{x}, \mathrm{y})$ sobre a projeção da VSS e a base interna da referida superfície para cada uma das duas cabeceiras do SBMT:

$$
\begin{aligned}
\mathrm{d}_{\text {VSS_CAB12 }} & ==\frac{|-5,904133 \cdot \mathrm{x}+1 \cdot \mathrm{y}-5 \cdot 439 \cdot 473,556|}{5,9882} \\
\mathrm{~d}_{\mathrm{VSS} \_C A B 30} & ==\frac{|-5,904133 \cdot \mathrm{x}+1 \cdot \mathrm{y}-5 \cdot 429 \cdot 177,336|}{5,9882}
\end{aligned}
$$

Seleção por atributo

Após a determinação de quais implantações encontram-se sobre a projeção da VSS, foi realizado processo de seleção por atributoatravés doArcEditor para identificar quais implantações são obstáculos à VSS. O fluxograma do processo está representado na Figura 11. 
Figura 11 - Fluxograma do processo de seleção por atributo.

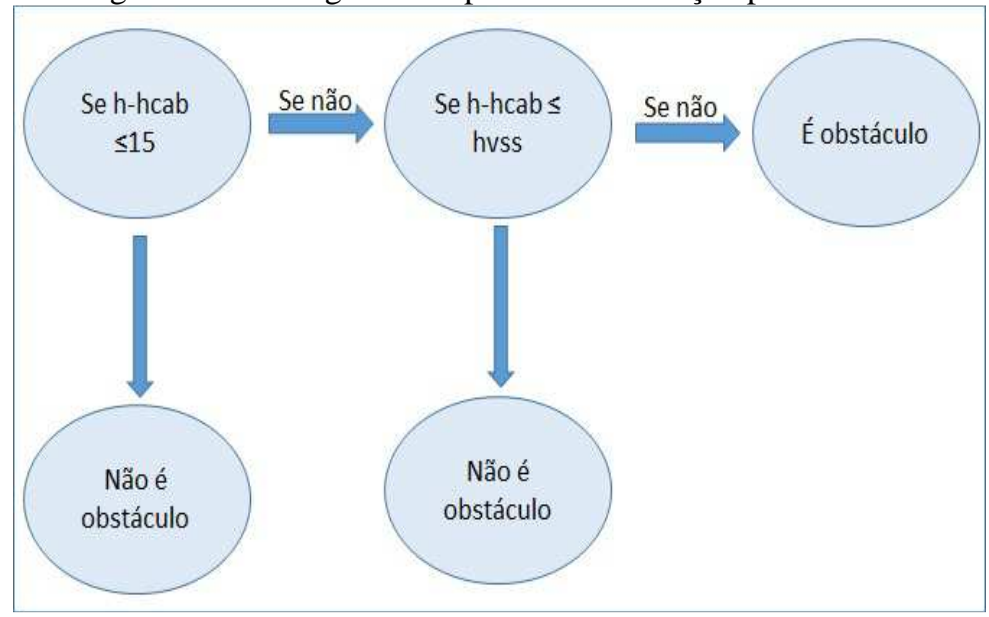

Em outras palavras, o processo de seleção por atributo deveriaidentificar as implantações que satisfizessem (9):

$$
\mathrm{h}-\mathrm{h}_{\mathrm{CAB}}>\mathrm{h}_{\mathrm{VSS}}>15 m
$$

onde:

- h é a elevação de topo da implantação;

- $\mathrm{h}_{\mathrm{CAB}}$ é a elevação da cabeceira a qual a VSS está associada;

- $h_{\text {VSS }}$ é a elevação da VSS sobre determinado ponto do plano xy.

A elevação $h_{\mathrm{V} s s}$ está representada em (10):

$$
\mathrm{h}_{\mathrm{VSS}}=\mathrm{d}_{\mathrm{VSS}} \cdot \operatorname{tg}\left(1,88^{\circ}\right)
$$

Onde $d_{V S S}$ é a distância no plano xy entre a implantação e a base da VSS, conforme (7) e (8).A Figura 12 apresenta os obstáculos à VSS dos procedimentos das duas cabeceiras:

Foram encontrados 19 obstáculos à VSS da cabeceira 12 e 11 obstáculos à VSS da cabeceira 30. Depois disso, verificou-se que, com exceção das antenas de celular e luminárias (postes), que possuem áreas pequenas para serem bem identificados na imagem, todos os obstáculos encontrados podem ser visualizados na imagem de satélite publicada no SIG que, conforme mencionado na seção 6.1, é datada de agosto de 2012. 
Figura 12 - Obstáculos a VSS do SBMT.

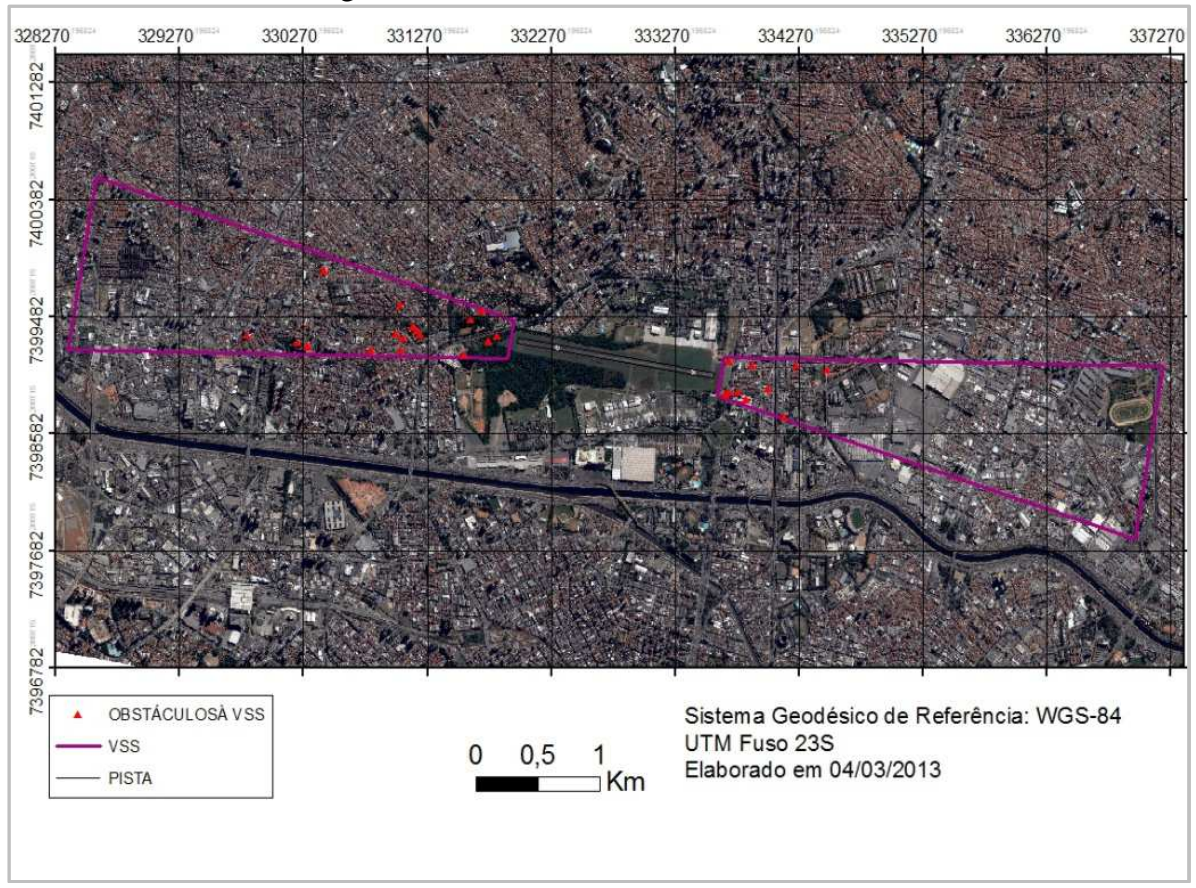

Algumas informações sobre os obstáculos, como suas coordenadas, altitude de topo e o quanto a VSS é violada por eles, estão apresentadas naTabela 1 e na Tabela 2.O Sistema Geodésico de Referência e o Datum Vertical são os mesmos do Cadastro de Implantações, descrito na seção 6.1.

A partir da Tabela 1, pode-se observar que há 7 obstáculos que violam em mais de $30 \mathrm{~m}$ a VSS do procedimento RNAV da cabeceira 12. Dentre eles, 3 são antenas de celular, 2 são casas, 1 é prédio e outro é torre de alta tensão. Além disso, dos 19 obstáculos, apenas um viola a VSS em menos de $10 \mathrm{~m}$.

Já a partir da Tabela 2, pode-se observar que o obstáculo que mais viola a VSS do procedimento RNAV da cabeceira 30 é a implantação 141 (Luminária), com 19,81 m de violação. Além disso, 9 dos 11 obstáculos violam a VSS em menos de $15 \mathrm{~m}$, sendo que 4 deles apresentam violação de menos de $10 \mathrm{~m}$.

Nesse sentido, com relação à VSS, é possível afirmar que há mais dificuldade para se implantar procedimento RNAV na cabeceira 12 do que na cabeceira 30, uma vez que a publicação do procedimento está associada à retirada ou rebaixamento dos obstáculos. 
Tabela 1 - Obstáculos à VSS do procedimento RNAV da cabeceira 12

\begin{tabular}{c|l|c|c|c|c|c}
\hline Número & \multicolumn{1}{|c|}{ Descrição } & $\begin{array}{c}\text { Altitude de } \\
\text { Topo }(\mathrm{m})\end{array}$ & $\begin{array}{c}\text { Coord.Norte } \\
(\mathrm{m})\end{array}$ & $\begin{array}{c}\text { Coord. Este } \\
(\mathrm{m})\end{array}$ & $\begin{array}{c}\text { Violação } \\
(\mathrm{m})\end{array}$ & Data Levantam. \\
\hline 17 & Bosque & 738,29 & 7399298,017 & 331764,165 & 10,78 & $13 / 08 / 2012$ \\
\hline 19 & Casa & 773,76 & 7399529,767 & 331719,115 & 43,53 & $13 / 08 / 2012$ \\
\hline 65 & Bosque & 749,92 & 7399197,336 & 331572,995 & 16,78 & $13 / 08 / 2012$ \\
\hline 68 & Prédio & 768,20 & 7399323,056 & 331083,764 & 18,54 & $13 / 08 / 2012$ \\
\hline 69 & Casa & 770,56 & 7399384,036 & 331188,274 & 23,94 & $13 / 08 / 2012$ \\
\hline 238 & Árvore & 781,11 & 7399462,882 & 331624,909 & 48,19 & $01 / 07 / 2009$ \\
\hline 239 & Alta Tensão & 783,81 & 7399405,182 & 331157,679 & 36,09 & $01 / 07 / 2009$ \\
\hline 240 & Casa & 766,86 & 7399348,231 & 331218,989 & 21,44 & $01 / 07 / 2009$ \\
\hline 241 & Antena de Celular & 780,08 & 7399358,642 & 331021,679 & 28,21 & $01 / 07 / 2009$ \\
\hline 242 & Antena de Celular & 789,14 & 7399230,801 & 330822,129 & 31,52 & $01 / 07 / 2009$ \\
\hline 243 & Casa & 765,98 & 7399232,011 & 331066,979 & 16,27 & $01 / 07 / 2009$ \\
\hline 258 & Casa & 782,74 & 7399575,172 & 331054,479 & 30,75 & $01 / 07 / 2009$ \\
\hline 259 & Antena de Celular & 809,29 & 7399836,042 & 330439,748 & 35,97 & $01 / 07 / 2009$ \\
\hline 263 & Árvore & 737,23 & 7399339,591 & 331834,939 & 11,79 & $01 / 07 / 2009$ \\
\hline 584 & Prédio & 797,51 & 7399266,607 & 330310,675 & 23,14 & $20 / 09 / 2010$ \\
\hline 592 & Prédio & 797,01 & 7399253,132 & 330308,144 & 22,62 & $20 / 09 / 2010$ \\
\hline 593 & Prédio & 790,08 & 7399291,181 & 330228,368 & 12,90 & $20 / 09 / 2010$ \\
\hline 594 & Prédio & 791,13 & 7399345,034 & 329816,447 & 0,33 & $20 / 09 / 2010$ \\
\hline 598 & Antena de Celular & 809,10 & 7399835,672 & 330441,874 & 35,85 & $20 / 09 / 2010$ \\
\hline
\end{tabular}

Tabela 2 - Obstáculos à VSS do procedimento RNAV da cabeceira 30

\begin{tabular}{c|l|c|c|c|c|c}
\hline Número & \multicolumn{1}{|c|}{ Descrição } & $\begin{array}{c}\text { Altitude de } \\
\text { Topo }(\mathrm{m})\end{array}$ & $\begin{array}{c}\text { Coord.Norte } \\
(\mathrm{m})\end{array}$ & $\begin{array}{c}\text { Coord. Este } \\
(\mathrm{m})\end{array}$ & $\begin{array}{c}\text { Violação } \\
(\mathrm{m})\end{array}$ & Data Levantam. \\
\hline 92 & Luminária & 742,51 & $7.399 .142,587$ & $333.709,468$ & 18,88 & $13 / 08 / 2012$ \\
\hline 111 & Casa & 741,40 & $7.399 .113,827$ & $333.896,588$ & 11,55 & $13 / 08 / 2012$ \\
\hline & $\begin{array}{l}\text { Subestação de } \\
\text { energia }\end{array}$ & 738,13 & $7.398 .934,047$ & $334.027,978$ & 3,05 & $13 / 08 / 2012$ \\
\hline 140 & Caixa d'água & 743,03 & $7.398 .849,421$ & $333.850,662$ & 13,22 & $01 / 07 / 2009$ \\
\hline 141 & Luminária & 743,81 & $7.398 .886,886$ & $333.677,488$ & 19,81 & $13 / 08 / 2012$ \\
\hline 180 & Antena de Celular & 748,40 & $7.399 .111,792$ & $334.258,713$ & 6,82 & $01 / 07 / 2009$ \\
\hline 186 & Conj. De Prédios & 751,03 & $7.399 .072,942$ & $334.501,803$ & 1,37 & $01 / 07 / 2009$ \\
\hline 190 & Caixa d'água & 743,00 & $7.398 .849,381$ & $333.851,472$ & 13,16 & $01 / 07 / 2009$ \\
\hline 200 & Antena de Celular & 749,05 & $7.398 .712,661$ & $334.154,363$ & 8,66 & $01 / 07 / 2009$ \\
\hline 333 & Árvore & 737,97 & $7.398 .903,871$ & $333.783,042$ & 10,65 & $01 / 07 / 2009$ \\
\hline 340 & Árvore & 737,31 & $7.398 .903,361$ & $333.714,842$ & 12,19 & $01 / 07 / 2009$ \\
\hline
\end{tabular}

\section{CONCLUSÕES}

A aplicação em SIG desenvolvida mostrou-se eficaz na análise de obstáculos à VSS, mesmo sem utilizar um pacote de ferramentas para análise 3D. A aplicação utilizada foi suficiente ao integrar análise espacial em duas dimensões com uma consulta baseada em conceitos de geometria analítica.

Além disso, a aplicação pode ser utilizada simultaneamente por diversos usuários, uma vez que os dados são publicados e acessíveis em ambiente web, diferente de aplicações em SIG desenvolvidos unicamente em ferramentas Desktop.

Bol. Ciênc. Geod., sec. Artigos, Curitiba, v. 20, nº 3, p.504-525, jul-set, 2014. 
Isso traz ganhos na medida em que usuários de setores diversos dentro de uma empresa operadora aeroportuária podem gerar os produtos cartográficos de que necessitam de forma descentralizada, sem necessitar requerê-los de outro setor.

Como limitação do trabalho, pode-se citar a utilização de cadastro de implantações proveniente de levantamentos ocorridos há mais de dois anos. Dos obstáculos encontrados, mesmo que a maioria deles tenha sido identificada na imagem de satélite, algumas implantações podem não mais existir hoje, outras podem ter sido rebaixadas, não se configurando mais como obstáculos, bem como outros novos obstáculos podem ter sido erguidos.

Diante disso, recomenda-se que a administração aeroportuária local realize atualização do levantamento topográfico para confirmar se as implantações descritas nas Tabelas 1 e 2 ainda existem. Caso as implantações ainda existam, faz-se necessário o levantamento de suas altitudes de topo. Com base nas altitudes de topo atuais e na metodologia apresentada neste trabalho será possível afirmar se as implantações continuam sendo obstáculos à VSS ou não.

Apesar dessa limitação, a metodologia para a determinação de qual implantação constante do cadastro é obstáculo provou-se eficaz.

Recomenda-se, para trabalhos futuros, a utilização de levantamentos LIDAR para a detecção das implantações no entorno do aeroporto, por meio do qualserá possível a identificação de uma grande quantidade de implantações de forma mais rápida que através de levantamento topográfico convencional.

Recomenda-se também, para trabalhos futuros, a utilização de ferramenta para análise espacial em três dimensões para a verificação de qual implantação é obstáculo à VSS.

\section{AGRADECIMENTOS}

O primeiro autor agradece à Infraero por propiciar a utilização dos softwares de SIG na elaboração deste trabalho.

\section{REFERÊNCIAS BIBLIOGRÁFICAS}

BENTES, F. M.; HELENO, T. A.; SLAMA, J. G. Analysis of airport noise exposure around Viracopos International Airport using geographic information systems. Journal of Air Transport Management, v. 31, p. 5-17, 2013.

BRASIL, Portaria N. ${ }^{\circ}$ 68/DGCEA, de 02 de maio de 2005. Aprova o Plano Específico de Zona de Proteção de Aeródromo de Porto Alegre.

BRASIL, Regulamento Brasileiro de Aviação Civil - RBAC 154, de 11 de maio de 2009. Projeto de aeródromos.

BRASIL, Portaria 256/GC5, de 11 de maio de 2011. Dispõe sobre as restrições relativas às implantações que possam afetar adversamente a segurança e a regularidade das operações aéreas, e dá outras providências.

DECEA (Departamento de Controle do Espaço Aéreo). Novas regras para aeroportos que operam por instrumentos. Disponível em http://www.fab. mil.br/portal/capa/index.php?mostra=10063. Acesso em 30 set. 2013. 
INFRAERO. Aeroporto de são Paulo - Campo de Marte. Disponível em http://www.infraero.gov.br/index.php/br/aeroportos/sao-paulo/aeroportocampo-de-marte.html. Acesso em 30 set. 2013.

INFRAERO.Estatísticas dos Aeroportos. Disponível em http://www.infraero.gov.br/index.php/br/estatistica-dos-aeroportos.html. Acesso em 14 mar. 2014.

HUANG, Z.; DAS, A.; QIU, Y.; TATEM, A. J. Web-based GIS: the vector-borne disease airline importation risk (VBD-AIR) tool. International Journal of Health Geographics 11:33, 2012.

ICAO (International Civil Aviation Organization). Doc 8168 OPS/611.Procedures for Air Navigation Services, Volume 2, Construction of Visual and Instrument Flight Procedures, Canada, 783p, 2006.

ICAO (International Civil Aviation Organization)(2008). Doc 9613 AN/937.Performance Based Navigation (PBN) Manual, Canada, 783p, 2008.

JIA, X.; CHUNG, D.; HUANG, J.; PETRILLI, M.; THE, L. ARO: Geographic Information System-Based System for optimizing airport runway orientation. Journal of Transportation Engineering, v. 130, p. 555-559, 2004.

MARTINS, J. L. F. Utilização de imagens de alta resolução para gerenciamento de zonas de proteção de aeródromos. Dissertação (Mestrado em Engenharia Cartográfica) - Universidade Federal do Rio Grande do Sul, 2010. 106p.

MEDEIROS, D. M. C. Sistemas de Aproximação RNAV e RNP AR - Estudo para aeroporto da Ilha do Pico. Dissertação (Mestrado em Engenharia Aeronáutica) - Universidade da Beira Interior, Covilhã, Portugal, 2011. 137p.

PARISH, C. E..Analysis of Airborne Laser-Scanning System Configurations for Detecting Airport Obstructions. Dissertação de mestrado - University of Florida, USA, 2003. 121p.

PARISH, C. E.; NOWAK, R. D. Improved Approach to LIDAR Airport Obstruction Surveying Using Full-Waveform Data.Journal of Surveying Engineering, v. 135, p. 72-82, 2009.

SANCHES, P. S.; BURGHOUWT, G.; BARBERA, M. P.An appraisal of the CORINE land cover database in airport catchment area analysis using a GIS approach. Journal of Air Transport Management, v. 34, p. 12-16, 2014.

SANTOS, G. S. Sistema de Informação Geográfica de Participação Pública aplicado à gestão do uso do solo urbano impactado pelo ruído aeronáutico. Dissertação (Mestrado em Engenharia de Infraestrutura Aeronáutica) Instituto Tecnológico de Aeronáutica, 2012.78p.

ZIMMER, N.; WIPPLINGER, P.; SCHIEFELE, J. Synthetic Generation of Airport Familiarization using Geo Information Systems. In: Remote Sensing for Environmental Monitoring, GIS Applications, and Geology VII. Proceedings of SPIE, v. 6749, 2007.

(Recebido em outubro de 2013. Aceito em março de 2014). 